\title{
Modern prevalence of the Fredrickson-Levy-Lees dyslipidemias: findings from the Very Large Database of Lipids and National Health and Nutrition Examination Survey
}

\author{
Vasanth Sathiyakumar ${ }^{1}$, Vincent A. Pallazola ${ }^{1}$, Jihwan Park ${ }^{2}$, Rachit M. Vakil', Peter P. Toth ${ }^{1,3}$, \\ Mariana Lazo-Elizondo ${ }^{2,4}$, Renato Quispe ${ }^{1,4}$, Eliseo Guallarr,4, Maciej Banach ${ }^{5}$, \\ Roger S. Blumenthal', Steven R. Jones ${ }^{1}$, Seth S. Martin ${ }^{1,4}$
}

${ }^{1}$ Ciccarone Center for the Prevention of Cardiovascular Disease, Division of Cardiology, Department of Medicine, Johns Hopkins University School of Medicine, Baltimore, MD, USA

2Department of Epidemiology, Johns Hopkins Bloomberg School of Public Health, Baltimore, MD, USA

${ }^{3}$ Department of Medicine, Community Hospital General Medical Center, Sterling, IL, USA ${ }^{4}$ Welch Center for Prevention, Epidemiology, and Clinical Research, Department of Epidemiology, Johns Hopkins Bloomberg School of Public Health, Baltimore, MD, USA ${ }^{5}$ Cardiovascular Research Centre, University of Zielona Gora, Zielona Gora, Poland

Submitted: 3 July 2019

Accepted: 4 July 2019

Arch Med Sci 2020; 16 (6): 1279-1287

DOI: https://doi.org/10.5114/aoms.2019.86964

Copyright (C) 2019 Termedia \& Banach

\section{Abstract}

Introduction: Five decades ago, Fredrickson, Levy, and Lees (FLL) qualitatively characterized clinical dyslipidemias with specific implications for cardiovascular and non-cardiovascular morbidity and mortality. They separated disorders of elevated cholesterol and triglycerides into five phenotypes (types I-V) based on their lipoprotein profile. Although clinicians generally consider them rare entities, modern FLL prevalence may be greater than previously reported.

Material and methods: We performed a cross-sectional analysis in 5,272 participants from the 2011-2014 National Health and Nutrition Examination Survey and 128,506 participants from the Very Large Database of Lipids study with complete, fasting lipid profiles. We used a validated algorithm to define FLL phenotypes employing apolipoprotein B, total cholesterol, and triglycerides.

Results: Overall prevalence of FLL phenotypes was 33.9\%. FLL prevalence in the general population versus clinical lipid database was: type I (0.05 vs. $0.02 \%$ ), type Ila (3.2 vs. $3.9 \%)$, type IIb (8.0 vs. $10.3 \%)$, type III (2.0 vs. $1.7 \%)$, type IV (20.5 vs. $24.1 \%)$, and type V (0.15 vs. $0.13 \%)$. Those aged $40-74$ years had a higher overall prevalence compared to other age groups $(p<0.001)$ and men had overall higher prevalence than women $(p<0.001)$. Those with diabetes $(51.6 \%)$ or obese BMI $(49.0 \%)$ had higher prevalence of FLL phenotypes compared to those without diabetes $(31.3 \% ; p<0.001)$ and normal BMI $(18.3 \% ; p<0.001)$

Conclusions: FLL phenotypes are likely far more prevalent than appreciated in clinical practice, in part due to diabetes and obesity epidemics. Given the prognostic and therapeutic importance of these phenotypes, their identification becomes increasingly important in the era of precision medicine.

Key words: Very Large Database of Lipids, National Health and Nutrition Examination Survey, hyperlipoproteinemia, apolipoprotein B, prevalence.
Corresponding author: Assoc. Prof. Seth S. Martin MD, MHS, FACC, FAHA, FASPC Advanced Lipids Disorders Program Ciccarone Center for the Prevention of Cardiovascular Disease Johns Hopkins Hospital $600 \mathrm{~N}$. Wolfe St Carnegie 591

21287 Baltimore, USA Phone: 410-502-0469

Facsimile: 410-367-2224 E-mail: smart100@jhmi.edu 


\section{Introduction}

Five decades ago, Fredrickson, Levy, and Lees (FLL) characterized clinical dyslipidemia phenotypes qualitatively using electrophoresis and ultracentrifugation [1]. They separated disorders of elevated cholesterol and/or triglycerides into five phenotypes (types I-V), each of which represented a characteristic abnormality: chylomicrons (types I, $\mathrm{V})$, very low-density lipoproteins (VLDL) (types IIb, IV, V), low-density lipoproteins (LDL) (types Ila, IIb), or remnant particles (type III). The World Health Organization (WHO) adopted this schema in 1972 [2].

Although modern lipid diagnosis has largely been simplified to identifying elevations in LDL-cholesterol (LDL-C) as reflected in worldwide guidelines, FLL phenotypes remain of prognostic and therapeutic importance [3-6]. Each phenotype has specific implications for atherosclerotic cardiovascular disease as VLDL and remnant particles are highly atherogenic $[7,8]$. Furthermore, these phenotypes are associated with non-cardiovascular morbidity including pancreatitis and xanthomata formation [9]. With the expanding armamentarium of lipid-lowering therapies aimed at specific lipoproteins, identifying the presence of a FLL phenotype may also facilitate personalized, targeted therapy [10-13].

In the era of FLL's seminal publications, lipid fractionation techniques were limited in their scalability to assess prevalence of these phenotypes in large studies [14]. As a result, the prevalence of FLL phenotypes has been uncertain and clinicians have generally viewed them as rare entitities [15-17]. However, the ongoing epidemics of overweight/obesity status and metabolic syndrome/ diabetes may affect the modern prevalence of FLL phenotypes, in particular those associated with an excess of triglyceride-rich lipoproteins [9, 18-20]. In addition, Sniderman et al. recently validated a quantitative approach to diagnose the FLL phenotypes integrating the conventional lipid profile with apolipoprotein B (apoB), a measure standardized by the $\mathrm{WHO}$ and readily available by most major laboratories worldwide [21, 22].

We therefore sought to determine the contemporary prevalence [15] of the FLL phenotypes in US adults in two settings: a national sample representative of the general population and a large clinical laboratory database reflecting individuals undergoing lipid testing in clinical practice.

\section{Material and methods}

\section{Study populations}

We examined fasting participants from the National Health and Nutritional Examination Survey (NHANES) 2011-2012 and 2013-2014 cycles, and fasting patients from the second harvest of the Very Large Database of Lipids (VLDL) study $[23,24]$. NHANES is a complex survey, conducted by the National Center for Health Statistics, to provide a representative sample of the civilian, non-institutionalized US population. The process of sample selection and participation in NHANES has been fully described previously [25]. This analysis included 5,272 fasting NHANES participants 18 years and older with complete lipid data (Supplementary Figure S1).

The VLDL study was constructed with de-identified data from the clinical operations of a single laboratory (VAP Diagnostics Lab, Birmingham, AL) [24]. The database includes patients from thousands of clinicians across the US in predominantly primary care settings. The process of de-identification and quality checks in the VLDL study was described elsewhere [24]. This cross-sectional analysis included 128,506 fasting patients 18 years and older from the VLDL study with apoB testing (Supplementary Figure S1). If a patient had more than one lipid test in the database, then we included only the first test in this analysis.

NHANES study protocols were approved by the National Center of Health Statistics institutional review board. Written informed consent was obtained from all participants. VLDL study protocols were reviewed by the Johns Hopkins IRB and deemed exempt due to the use of de-identified data only.

\section{Lipid measurements}

Within NHANES, total cholesterol, high-density lipoprotein-cholesterol (HDL-C), and triglycerides (TG) were measured photometrically using oxidation reagents. $\mathrm{LDL}-\mathrm{C}$ and VLDL-cholesterol (VLDL-C) were calculated and apoB was measured through immunochemical reactions and light spectroscopy [26-28]. The full process of lipid measurement, data checking, and quality inspection within NHANES has been documented in detail elsewhere [26-28].

In the VLDL dataset, Vertical Auto Profile (VAP) methodology (VAP Diagnostics Lab, Inc., Birmingham, AL) was used to directly measure the cholesterol content in LDL, VLDL, HDL, and intermediate-density lipoproteins (IDL) [24]. The VAP method uses single vertical spin density gradient ultracentrifugation to separate lipoprotein fractions in less than $1 \mathrm{~h}$. The cholesterol contents of the eluted lipoprotein fractions were measured using a validated colorimetric assay employing cholesterol oxidase reactions. TG values were directly measured with the Abbott ARCHITECT C-8000 system (Abbott Laboratories, Abbott Park, IL). Yearly quality assessments with VAP and Abbott ARCHITECT-derived values have been described previously [24]. ApoB was directly 
measured through a WHO standardized immunoassay based on immunoturbidimetry with the Abbott ARCHITECT analyzer and reagent.

\section{Definitions of dyslipidemia}

We defined conventional dyslipidemia using cutpoints set forth by the National Cholesterol Education Program's Adult Treatment Panel III guidelines for elevated LDL-C at two targets: $\geq 160 \mathrm{mg} / \mathrm{dl}$ and $\geq 130 \mathrm{mg} / \mathrm{dl}$ (Figure 1) [3]. In this study, we used the Sniderman algorithm to define the FLL phenotypes, which uses two values from a standard lipid profile (total cholesterol and TG) in conjunction with apoB levels (Figure 1) [21]. This algorithm was derived from a cohort of 1,771 fasting patients with genetically verified dyslipidemias and has been validated using other cohorts [21, 22].

\section{Other variables}

In NHANES, demographic and prescription medication information was gathered via in-home interviews and questionnaires [23, 25]. Race categories included: non-Hispanic White, non-Hispanic Black, Mexican American, and other. NHANES defined lipid-lowering therapies as the following: statins, bile acid sequestrants, and cholesterol absorption inhibitors. Body mass index (BMI) was calculated via height and weight measurements, and was categorized as: underweight $(<18.5)$, normal ( $\geq 18.5$ to $<25)$, overweight $(\geq 25$ to $<30)$, and obese $(\geq 30)$. Diabetes was assessed via measurement of fasting blood glucose and hemoglobin $A_{1 c}$, defined as: fasting glucose $\geq 126 \mathrm{mg} / \mathrm{dl}$, hemoglobin $A_{1 c} \geq 6.5 \%$, or self-report of a previous physician diagnosis. In the VLDL study, additional variables included age and sex.

\section{Statistical analysis}

In NHANES, we accounted for the complex sampling design and used sampling weights to generate nationally representative estimates in all analyses. We used the Taylor series (linearization) method to calculate standard errors $[23,25]$. In the VLDL study, we compared distributions of lipid parameters in participants with available apoB values and those without apoB values. In each study, we examined the prevalence for the overall study populations and stratified by age and sex. Comparisons of prevalence were made using $\chi^{2}$ tests. We calculated medians and interquartile ranges (IQR) for each lipid parameter and by phenotype. We produced kernel density plots for pertinent lipid values. We also used logistic regression in NHANES participants to determine FLL phenotype prevalence by clinical characteristics such as BMI and diabetes marginally adjusted for age, sex, and

A

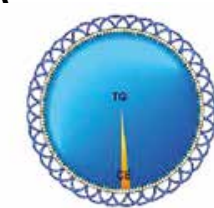

Type I: Chylomicrons apoB $<75 \mathrm{mg} / \mathrm{dl}$, $\mathrm{TG} \geq 133 \mathrm{mg} / \mathrm{dl}$, $\mathrm{TG} / \mathrm{apoB} \geq 10$

Type Ila: LDL

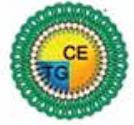
apoB $\geq 120 \mathrm{mg} / \mathrm{dl}$, $\mathrm{TG}<133 \mathrm{mg} / \mathrm{dl}$

Type IIb: LDL + VLDL apoB $\geq 120 \mathrm{mg} / \mathrm{dl}$, $\mathrm{TG} \geq 133 \mathrm{mg} / \mathrm{dl}$

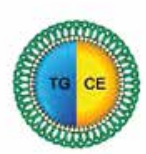

Type III: Remnants apoB $<120 \mathrm{mg} / \mathrm{dl}$, $\mathrm{TG} \geq 133 \mathrm{mg} / \mathrm{dl}$, TG/apoB $<8.8, T C /$ apoB $\geq 2.4$

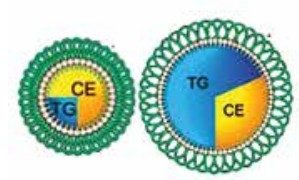

Type IV: VLDL apoB $<120 \mathrm{mg} / \mathrm{dl}$, $\mathrm{TG} \geq 133 \mathrm{mg} / \mathrm{dl}$, TG/apoB $<8.8$, TC/ароB $<2.4$
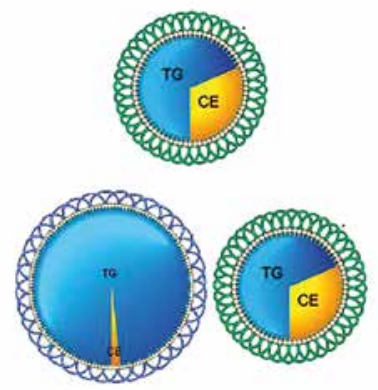

Type V: Chylomicrons + VLDL $a p o B \geq 75$ and $<120 \mathrm{mg} / \mathrm{dl}$, TG $\geq 133 \mathrm{mg} / \mathrm{dl}$, TG/apoB $\geq 8.8$

B

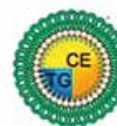

Cutpoint 1

LDL-C $\geq 130 \mathrm{mg} / \mathrm{dl}$

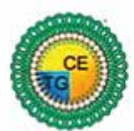

Cutpoint 2

LDL-C $\geq 160 \mathrm{mg} / \mathrm{dl}$

Figure 1. Dyslipidemia classification schema. A - Fredrickson-Levy-Lees classification for types I-V using the apoB algorithm. B - Conventional criteria classification using only LDL-C at two guideline-recommended cutpoints

$C E$ - cholesterol ester, TG - triglyceride, $L D L$ - low-density lipoprotein, VLDL - very low-density lipoprotein, apoB - apolipoprotein B, LDL-C - low-density lipoprotein-cholesterol. 
race/ethnicity. All statistical tests were two-sided with significance at $p<0.05$. Statistical analyses were conducted using Stata statistical software version 14.2 (StataCorp, College Station, TX).

\section{Results}

\section{Study populations}

The 5,272 NHANES participants had a median age of 45 years (IQR: $32-60$ years), with $48.4 \%$ men (Table I). The majority of NHANES participants were overweight or obese (68.8\%). Nearly

Table I. Characteristics of NHANES and VLDL study populations

\begin{tabular}{|c|c|c|}
\hline Parameter & $\begin{array}{l}\text { NHANES }^{a} \\
(n=5,272)\end{array}$ & $\begin{array}{c}\operatorname{VLDL}^{\mathrm{b}} \\
(n=128,506)\end{array}$ \\
\hline \multicolumn{3}{|l|}{ Demographics: } \\
\hline $\begin{array}{l}\text { Age, median (IQR) } \\
\text { [years] }\end{array}$ & $45(32-60)$ & $57(46-68)$ \\
\hline Men, $n(\%)$ & $2,578(48.4)$ & $57,221(44.8)$ \\
\hline \multicolumn{3}{|l|}{ Race, $n(\%)$ : } \\
\hline Non-Hispanic white & $2,173(66.2)$ & \\
\hline Non-Hispanic black & $1,132(11.6)$ & \\
\hline Mexican American & $632(8.6)$ & \\
\hline Other & $1,335(13.7)$ & \\
\hline \multicolumn{3}{|l|}{ BMI category, $n(\%)$ : } \\
\hline Underweight & $107(1.7)$ & \\
\hline Normal & $1,610(29.5)$ & \\
\hline Overweight & $1,639(32.2)$ & \\
\hline Obese & $1,916(36.5)$ & \\
\hline $\begin{array}{l}\text { Presence of diabetes, } \\
n(\%)\end{array}$ & $906(12.8)$ & \\
\hline $\begin{array}{l}\text { Lipid-lowering therapy, } \\
n(\%) \text { : }\end{array}$ & $1,058(33.4)$ & \\
\hline Statin use & $981(31.4)$ & \\
\hline \multicolumn{3}{|c|}{ Lipid characteristics, median (IQR) [mg/dl]: } \\
\hline Total cholesterol & $187(162-214)$ & $197(168-228)$ \\
\hline ApoB & $87(72-105)$ & $92(76-108)$ \\
\hline HDL-C & $51(43-62)$ & $52(43-64)$ \\
\hline VLDL-C & $20(16-26)$ & $22(17-29)$ \\
\hline IDL-Cc & & $13(9-19)$ \\
\hline LDL-C & $111(89-136)$ & $116(92-144)$ \\
\hline TG & 99 (68-147) & $110(78-159)$ \\
\hline
\end{tabular}

aIn NHANES, estimated percentages are weighted to reflect survey procedures. ${ }^{b}$ Information on race, BMI, co-morbidities and medication use was not available in the VLDL dataset. $I D L-C$ was not measured in NHANES. ApoB - apolipoprotein B, HDL-C - high-density lipoproteincholesterol, VLDL-C - very low-density lipoprotein-cholesterol, IDL-C - intermediate density lipoprotein-cholesterol, LDL-C - low-density lipoprotein-cholesterol, TG - triglyceride, $\mathrm{mg} / \mathrm{dl}$ - milligrams per deciliter.
1 in 3 NHANES participants were on lipid-lowering therapies, predominately statins. The median age of the 128,506 VLDL study patients was 57 years (IQR: 46-68 years), with $44.8 \%$ men, similar to the age (median: 57 years; IQR: 47-67 years) and sex distributions (47.4\% men) in VLDL patients without apoB values. Furthermore, lipid distributions and percentiles of total cholesterol, LDL-C, HDL-C, and TG were similar between included VLDL patients compared to patients in the database without apoB values (Supplementary Figure S2).

\section{Overall FLL phenotype prevalence}

Using conventional criteria, the proportions of participants with LDL-C $\geq 160 \mathrm{mg} / \mathrm{dl}$ in NHANES and in the VLDL study were $9.5 \%$ and $14.9 \%$, respectively. The corresponding proportions when using LDL-C $\geq 130 \mathrm{mg} / \mathrm{dl}$ were $30.3 \%$ and $37.2 \%$, respectively. A greater percentage of individuals was categorized as dyslipidemic by the FLL criteria compared to the conventional criteria $(p<0.001)$. The proportions of individuals with one of the FLL phenotypes were $33.9 \%$ in NHANES and $40.3 \%$ in the VLDL study. Among NHANES participants with LDL-C $<130 \mathrm{mg} / \mathrm{dl}$ or $<160 \mathrm{mg} / \mathrm{dl}$, the proportions of participants with one of the FLL phenotypes were $16.9 \%$ and $25.9 \%$, respectively. Corresponding proportions in the VLDL study were 19.6 and $28.8 \%$, respectively.

\section{Individual FLL phenotype prevalence}

By individual FLL phenotype, type IV dyslipidemia was the most prevalent in both NHANES and VLDL (prevalence of $20.5 \%$ and $24.1 \%$, respectively; Table II), followed by type IIb (prevalence of $8.0 \%$ and $10.3 \%$, respectively). Types IIa and III phenotypes were present in $3-4 \%$ and $2 \%$ of participants in both studies, respectively, while types $\mathrm{I}$ and $\mathrm{V}$ were rare in both studies.

\section{FLL phenotype prevalence by clinical characteristics}

The patterns of FLL phenotypes by age and sex groups were similar to that in the overall sample (Table II), although participants 40-74 years of age had a higher overall prevalence of the FLL phenotypes, particularly types IIa, IIb, and IV, compared to other age groups $(p<0.001)$. Men had greater overall prevalence of the phenotypes compared to women, especially with types IIb and IV ( $p<0.001)$.

Using NHANES data, we further evaluated the marginally adjusted prevalence of FLL phenotypes by race, BMI, and presence of diabetes (Figure 2, Supplementary Table SI). By race, the prevalence of participants with one of the FLL phenotypes was lowest in non-Hispanic Blacks and highest in Mexican Americans (20.5\% vs. $42.7 \%$; $p<0.001$ ). 
Modern prevalence of the Fredrickson-Levy-Lees dyslipidemias: findings from the Very Large Database of Lipids and National Health and Nutrition Examination Survey

Table II. Prevalence, \% $(95 \% \mathrm{CI})$ of the FLL phenotypes in NHANES and VLDL study populations

\begin{tabular}{|c|c|c|c|c|c|c|c|c|}
\hline Variable & Normal & Any FLL & Type I & Type Ila & Type IIb & Type III & Type IV & Type V \\
\hline \multicolumn{9}{|l|}{ NHANES: } \\
\hline Overall & $\begin{array}{c}66.1 \\
(63.9-68.2)\end{array}$ & $\begin{array}{c}33.9 \\
(31.8-36.1)\end{array}$ & $\begin{array}{c}0.05 \\
(0.01-0.36)\end{array}$ & $\begin{array}{c}3.2 \\
(2.5-4.1)\end{array}$ & $\begin{array}{c}8.0 \\
(6.9-9.3)\end{array}$ & $\begin{array}{c}2.0 \\
(1.5-2.6)\end{array}$ & $\begin{array}{c}20.5 \\
(18.6-22.6)\end{array}$ & $\begin{array}{c}0.15 \\
(0.06-0.43)\end{array}$ \\
\hline \multicolumn{9}{|l|}{ Age [years]: } \\
\hline $18-39$ & $\begin{array}{c}76.0 \\
(73.1-78.7)\end{array}$ & $\begin{array}{c}24.0 \\
(31.3-26.9)\end{array}$ & $\begin{array}{c}0.12 \\
(0.02-0.93)\end{array}$ & $\begin{array}{c}2.0 \\
(1.4-2.9)\end{array}$ & $\begin{array}{c}5.0 \\
(3.8-6.6)\end{array}$ & $\begin{array}{c}1.6 \\
(1.0-2.6)\end{array}$ & $\begin{array}{c}15.1 \\
(13.1-17.3)\end{array}$ & $\begin{array}{c}0.17 \\
(0.05-0.63)\end{array}$ \\
\hline $40-74$ & $\begin{array}{c}59.1 \\
(55.7-62.5) \\
\end{array}$ & $\begin{array}{c}40.9 \\
(37.5-44.3)\end{array}$ & - & $\begin{array}{c}4.1 \\
(3.2-5.3)\end{array}$ & $\begin{array}{c}10.5 \\
(8.7-12.5)\end{array}$ & $\begin{array}{c}2.1 \\
(1.4-3.2) \\
\end{array}$ & $\begin{array}{c}24.0 \\
(20.6-27.9)\end{array}$ & $\begin{array}{c}0.16 \\
(0.03-0.84)\end{array}$ \\
\hline$\geq 75$ & $\begin{array}{c}66.0 \\
(61.8-69.8)\end{array}$ & $\begin{array}{c}34.1 \\
(30.2-38.2)\end{array}$ & - & $\begin{array}{c}2.8 \\
(1.4-5.4)\end{array}$ & $\begin{array}{c}5.2 \\
(3.1-8.5)\end{array}$ & $\begin{array}{c}2.8 \\
(1.0-7.2)\end{array}$ & $\begin{array}{c}23.3 \\
(19.2-28.0)\end{array}$ & - \\
\hline \multicolumn{9}{|l|}{ Sex: } \\
\hline Men & $\begin{array}{c}61.2 \\
(58.2-64.0)\end{array}$ & $\begin{array}{c}38.9 \\
(36.0-41.8)\end{array}$ & $\begin{array}{c}0.10 \\
(0.01-0.74)\end{array}$ & $\begin{array}{c}3.1 \\
(2.4-4.2)\end{array}$ & $\begin{array}{c}9.1 \\
(7.6-10.8)\end{array}$ & $\begin{array}{c}1.9 \\
(1.3-2.8)\end{array}$ & $\begin{array}{c}24.4 \\
(21.6-27.3)\end{array}$ & $\begin{array}{c}0.28 \\
(0.08-0.94)\end{array}$ \\
\hline Women & $\begin{array}{c}70.7 \\
(68.6-72.7)\end{array}$ & $\begin{array}{c}29.3 \\
(27.3-31.4)\end{array}$ & - & $\begin{array}{c}3.3 \\
(2.4-4.5)\end{array}$ & $\begin{array}{c}7.0 \\
(5.7-8.5)\end{array}$ & $\begin{array}{c}2.0 \\
(1.4-2.9)\end{array}$ & $\begin{array}{c}17.0 \\
(15.2-18.9)\end{array}$ & $\begin{array}{c}0.03 \\
(0.00-0.26)\end{array}$ \\
\hline \multicolumn{9}{|l|}{ VLDL study: } \\
\hline Overall & $\begin{array}{c}59.8 \\
(59.5-60.0)\end{array}$ & $\begin{array}{c}40.3 \\
(40.0-40.5)\end{array}$ & $\begin{array}{c}0.02 \\
(0.02-0.04)\end{array}$ & $\begin{array}{c}3.9 \\
(3.8-4.0)\end{array}$ & $\begin{array}{c}10.3 \\
(10.2-10.5)\end{array}$ & $\begin{array}{c}1.7 \\
(1.7-1.8)\end{array}$ & $\begin{array}{c}24.1 \\
(23.9-24.4)\end{array}$ & $\begin{array}{c}0.13 \\
(0.11-0.15)\end{array}$ \\
\hline \multicolumn{9}{|l|}{ Age [years]: } \\
\hline $18-39$ & $\begin{array}{c}67.7 \\
(67.0-68.4)\end{array}$ & $\begin{array}{c}32.3 \\
(31.7-33.0)\end{array}$ & $\begin{array}{c}0.04 \\
(0.02-0.09)\end{array}$ & $\begin{array}{c}3.1 \\
(2.9-3.4)\end{array}$ & $\begin{array}{c}8.2 \\
(7.8-8.6)\end{array}$ & $\begin{array}{c}1.3 \\
(1.2-1.5)\end{array}$ & $\begin{array}{c}19.5 \\
(18.9-20.1)\end{array}$ & $\begin{array}{c}0.18 \\
(0.13-0.26)\end{array}$ \\
\hline $40-74$ & $\begin{array}{c}57.2 \\
(56.8-57.5) \\
\end{array}$ & $\begin{array}{c}42.8 \\
(42.5-43.2) \\
\end{array}$ & $\begin{array}{c}0.02 \\
(0.02-0.04)\end{array}$ & $\begin{array}{c}4.3 \\
(4.2-4.5) \\
\end{array}$ & $\begin{array}{c}11.6 \\
(11.4-11.8) \\
\end{array}$ & $\begin{array}{c}1.8 \\
(1.7-1.9) \\
\end{array}$ & $\begin{array}{c}25.0 \\
(24.7-25.3) \\
\end{array}$ & $\begin{array}{c}0.14 \\
(0.11-0.16) \\
\end{array}$ \\
\hline$\geq 75$ & $\begin{array}{c}65.0 \\
(64.3-65.7) \\
\end{array}$ & $\begin{array}{c}35.0 \\
(34.3-35.7)\end{array}$ & $\begin{array}{c}0.01 \\
(0.00-0.04)\end{array}$ & $\begin{array}{c}2.3 \\
(2.1-2.6) \\
\end{array}$ & $\begin{array}{c}6.3 \\
(5.9-6.6) \\
\end{array}$ & $\begin{array}{c}2.0 \\
(1.8-2.2) \\
\end{array}$ & $\begin{array}{c}24.4 \\
(23.8-25.0)\end{array}$ & $\begin{array}{c}0.01 \\
(0.00-0.04)\end{array}$ \\
\hline \multicolumn{9}{|l|}{ Sex: } \\
\hline Men & $\begin{array}{c}55.6 \\
(55.2-56.0)\end{array}$ & $\begin{array}{c}44.4 \\
(44.4-44.8)\end{array}$ & $\begin{array}{c}0.04 \\
(0.03-0.06)\end{array}$ & $\begin{array}{c}3.6 \\
(3.4-3.7)\end{array}$ & $\begin{array}{c}11.0 \\
(10.8-11.3)\end{array}$ & $\begin{array}{c}1.1 \\
(1.0-1.2)\end{array}$ & $\begin{array}{c}28.4 \\
(28.1-28.8)\end{array}$ & $\begin{array}{c}0.21 \\
(0.18-0.25)\end{array}$ \\
\hline Women & $\begin{array}{c}63.1 \\
(62.7-63.5)\end{array}$ & $\begin{array}{c}36.9 \\
(36.6-37.3)\end{array}$ & $\begin{array}{c}0.01 \\
(0.00-0.02)\end{array}$ & $\begin{array}{c}4.1 \\
(4.0-4.3)\end{array}$ & $\begin{array}{c}9.8 \\
(9.6-10.0)\end{array}$ & $\begin{array}{c}2.3 \\
(2.2-2.4)\end{array}$ & $\begin{array}{c}20.7 \\
(20.4-21.0)\end{array}$ & $\begin{array}{c}0.06 \\
(0.04-0.08)\end{array}$ \\
\hline
\end{tabular}

CI - confidence interval, NHANES - National Health and Nutrition Examination Survey, VLDL - Very Large Database of Lipids, FLL - Fredrickson-Levy-Lees.

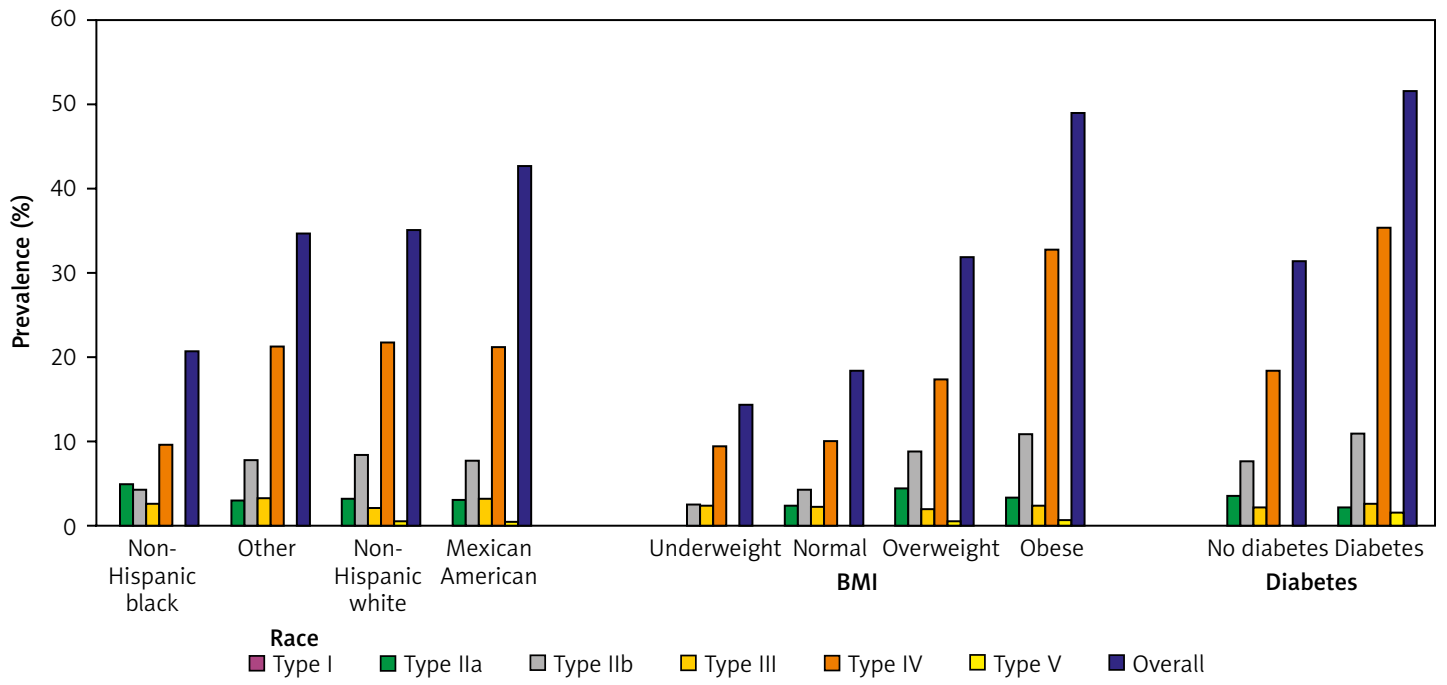

Figure 2. FLL phenotype prevalence by race, BMI, and diabetes

BMI - body mass index (underweight: <18.5; normal: $\geq 18.5$ and <25; overweight: $\geq 25$ and < 30; obese: $\geq 30$ ). Prevalence by race was marginally adjusted for age and sex, while prevalences by BMI and diabetes were marginally adjusted for age, sex, and race. 
The obese group had nearly triple the prevalence, and overweight group had nearly double the prevalence of individuals with any FLL phenotype compared to the normal BMI group $(p<0.001)$. The diabetes group had nearly double the prevalence of persons with one of the FLL phenotypes compared to participants without diabetes $(p<0.001)$.

\section{Lipid values}

The median levels of total cholesterol, apoB, and TG in NHANES participants without any FLL phenotype were 178,80 , and $79 \mathrm{mg} / \mathrm{dl}$, respectively. The corresponding medians in the VLDL study were 188, 83, and $85 \mathrm{mg} / \mathrm{dl}$, respectively (Supplementary Table SII and Supplementary Figure S3). Individuals with any FLL phenotype in NHANES had median total cholesterol values ranging from 189-253 mg/dl, apoB values of $69-134 \mathrm{mg} / \mathrm{dl}$, and TG values of $171-1,311 \mathrm{mg} / \mathrm{dl}$. The respective ranges in VLDL patients were 149-268 mg/dl, 64-134 mg/dl, and 106-1,143 mg/dl.

The median levels of lipid parameters in each FLL phenotype were similar in both studies, with expected elevations in the pertinent parameters characteristic for each phenotype. For example, in type IV, median VLDL-C values were $30 \mathrm{mg} / \mathrm{dl}$ for both NHANES and VLDL populations. In contrast, participants in NHANES with the type Ilb phenotype had similar elevations in VLDL-C $(36 \mathrm{mg} / \mathrm{dl})$ but also had median LDL-C values of $168 \mathrm{mg} / \mathrm{dl}$. The corresponding medians in Type IV VLDL study patients were 38 and $171 \mathrm{mg} / \mathrm{dl}$, respectively.

\section{Discussion}

The key message of our study is that in contemporary samples, the FLL lipoprotein phenotypes of diagnostic, prognostic, and therapeutic relevance appear far more prevalent than historically and clinically appreciated. This is especially true for the type IV phenotype which was present in approximately $20 \%$ of the study populations, as well as for rarer phenotypes such as the type III which was found in $2 \%$ of participants. A similar high prevalence of the FLL phenotypes was found in a representative sample of the US population and in a large clinical laboratory sample representing predominately primary care patients.

\section{Historical trends}

Smaller population studies using electrophoresis and ultracentrifugation previously suggested a low prevalence of certain phenotypes [15-17]. Reported frequencies of the type IIb phenotype ranged from $2-4 \%$, and type III was postulated to occur in $<0.7 \%$ of individuals, with prevalence as low as $0.01 \%$ depending on the population $[15-17,29,30]$. In our study, the type Ilb pheno- type was 2-5 times as prevalent, and the type III phenotype 3-4 times as prevalent as previously reported. Both phenotypes are highly atherogenic. The type lla phenotype, typically associated with familial hypercholesterolemia, also appears to be more common than formerly appreciated. The prevalence of familial hypercholesterolemia is estimated at 1 in 250 (0.4\%; or more than 1 million cases in the US) [31, 32]. Yet, the type Ila phenotype had a prevalence nearly 10 times greater (3-4\%), likely reflecting non-familial hypercholesterolemia cases. In contrast, the types $\mathrm{I}$ and $\mathrm{V}$ phenotypes appear to be truly rare, each with a prevalence well below $0.5 \%$ as suggested in prior reports [9, 15-17, 33].

The overall high FLL phenotype prevalence, especially types IIb and IV, may be the lipid manifestations of the obesity and diabetes epidemics as these conditions result in hypersecretion of hepatic VLDL $[18,34]$. In fact, this was a trend predicted by Fredrickson, Levy, and Lees in their seminal papers [11]. Despite a lack of large-scale databases at the time, the authors postulated that type IV was likely the most prevalent in part due to a large number of secondary causes, particularly diabetes. Nearly 1 in every 3 adult Americans have prediabetes or diabetes and approximately 2 in every 3 are overweight or obese [35]. While smaller cohort studies have suggested a relatively high prevalence of type IV dyslipidemia in specialized populations, our study demonstrates the very high prevalence of this phenotype across age and sex in the general population and its correlation with diabetes and obesity [36].

Our analysis included individuals both on and off lipid-lowering therapies. Lipid-lowering agents may convert patients from one phenotype to another (i.e., from a type IIb to type IV with statin therapy) or to the "normal" category. Given the high prevalence of statin use in the US, our results may underrepresent the true prevalence of the FLL phenotypes, particularly types IIa and IIb phenotypes characterized by excess LDL-C. However, since lipid-lowering drugs have become standardof-care and part of the natural history of dyslipidemia, our prevalence results are representative of how clinicians may encounter and manage dyslipidemia [37].

\section{Implications for patient-care}

Although the conventional approach of using LDL-C has been the focal point in clinical guidelines, the FLL phenotypes carry implications for morbidity and mortality and may improve dyslipidemia management. First, the majority of the lipoproteins in the phenotypes are atherogenic and associated with increased cardiovascular risk [7, 8, 38, 39]. Identifying FLL phenotypes is an oppor- 
tunity to manage primary or residual cardiovascular risk in patients that appear normal under the conventional approach of only using LDL-C. This is especially important for patients with clinical or advanced subclinical atherosclerotic cardiovascular disease [40]. In isolation, LDL-C does not identify atherogenic triglyceride-enriched lipoproteins such as VLDL and remnant particles. Our data show that FLL dyslipidemias are present in $17-20 \%$ of individuals with LDL-C $<130 \mathrm{mg} / \mathrm{dl}$, which highlights the opportunity to expand lipid management to lipoproteins beyond LDL.

Second, these phenotypes are further associated with non-cardiovascular morbidity, including life-threatening pancreatitis and pancreatic insufficiency, dermatologic manifestations (i.e. eruptive xanthomata and tendinous xanthomata), and ophthalmologic lesions (i.e. lipemia retinalis and corneal arcus) [1, 9]. These morbidities are only definitively treated by recognizing and addressing the underlying lipid disorder. The FLL phenotypes may subsequently present a framework to investigate underlying dyslipidemia in the presence of one of these clinical findings.

Third, these phenotypes are often genetic or hereditary as suggested by prior familial analyses and genetic studies $[1,9]$. Although the phenotypes may be secondary to systemic conditions such as renal dysfunction and thyroid dysregulation, several of these phenotypes may be primary in nature and directly linked to deficiencies in proteins and/or enzymes involved in lipoprotein metabolism, including lipoprotein lipase, apolipoprotein CII, and apolipoprotein E among others [9, $41,42]$. Diagnosing the presence of a phenotype in an index family member may therefore help identify potential genetic causes and facilitate timely detection and treatment in other family members.

Fourth, with further lipoprotein-lowering interventions in development, dyslipidemia treatment may improve by evolving to a more personalized approach that targets specific lipoproteins. For example, in type Ila dyslipidemia, LDL is in excess and statins are the clear first line of therapy. However, in type III dyslipidemia, the excess is in TG-rich remnants and therefore statins are not clearly effective, but fibrates, omega-3 derivatives, or niacin could be indicated. More potent, novel agents in development include targets against apolipoprotein CIII and angiopoietin-like 3 and 4 to specifically affect enzymes such as lipoprotein lipase and preferentially decrease TG-rich lipoproteins [12, 43]. FLL phenotyping may consequently aid in patient selection for the selective use of current and emerging dyslipidemia therapies to reduce lifetime cumulative exposure to atherogenic lipoproteins.

\section{Limitations and strengths}

We recognize limitations of our study. The VLDL population specifically does not have information on several co-morbidities which may be associated with the FLL phenotypes. This restricted the assessment of clinical associations such as diabetes and obesity to NHANES participants.

In both study populations, chylomicrons were not directly isolated and were presumably reflected in the VLDL-C fraction. Genetic analyses and family histories for individuals were not available, limiting our assessment of primary versus secondary causes of the phenotypes. Nevertheless, given that treatment is directed at phenotypic expression of dyslipidemia, our results are clinically relevant. We further restricted our analysis to individuals 18 years of age or older to provide prevalence estimates specifically for adults. Children and adolescents may be more likely to have a primary dyslipidemia and warrant separate analysis. Our study was also restricted to individuals with fasting lipid profiles because this has been a common standard for diagnosing primary dyslipidemias and because the diagnostic algorithm that we used was derived and validated in fasting samples. The generalizability to non-fasting lipid samples, which are of increasing clinical interest, should be considered in future studies.

Strengths of our study include the complementary, cross-sectional nature of our analysis, as our results are indicative of the FLL prevalence in both the general population and those specifically referred for clinical lipid testing. To our knowledge, our study is also the first to use a validated, scalable algorithm to diagnose the FLL phenotypes in big data samples - limitations which previously prevented accurate prevalence estimation. The algorithm only requires components that are standardized by the Centers for Disease Control and Prevention (CDC) and the WHO. Specifically, the CDC Lipids Reference Laboratory provides reference values for total cholesterol and TG, whereas apoB values are provided by the Northwest Lipid Metabolism and Diabetes Research Laboratories. This standardization allows for widespread and scalable phenotyping across various clinical and laboratory settings.

In conclusion, the FLL dyslipidemia phenotypes, which are endorsed by the WHO, are likely much more prevalent than historically and clinically appreciated. This may be explained in part by the lack of large-scale studies on this topic previously as well as by the epidemics of obesity and diabetes. The high prevalence of the FLL phenotypes has important diagnostic, prognostic, and therapeutic implications. Since FLL dyslipidemia diagnosis can be automated and scaled, these results open the door for a precision medicine approach in dyslipidemia management to reduce athero- 
sclerotic cardiovascular disease and non-cardiovascular morbidity.

\section{Acknowledgments}

The Very Large Database of Lipids has received support from the David and June Trone Family Foundation. Dr. Martin has received research support from the PJ Schafer Cardiovascular Research Fund, American Heart Association, Aetna Foundation, CASCADE FH, Maryland Innovation Initiative, iHealth, Stanford MedX, Nokia, Google, and Apple.

\section{Conflict of interest}

VS, VAP, JP, RMV, MLE, RQ, EG, MB, and RSB have no relevant disclosures. PPT: Speakers Bureau; Amarin, Akcea, Amgen, Kowa, Merck, Nova Nordisk, Regeneron, Sanofi (Consultant/Advisory Board). SRJ: Co-inventor for a method to estimate LDL cholesterol levels, patent application pending; funding from the David and June Trone Family Foundation. SSM: Co-inventor for a method to estimate LDL cholesterol levels, patent application pending; Consultant/Advisory Board for Sanofi/ Regeneron, Amgen, Quest Diagnostics, Akcea, Novo Nordisk, Esperion.

\section{References}

1. Fredrickson DS, Levy RI, Lees RS. Fat transport in lipoproteins: an integrated approach to mechanisms and disorders. N Engl J Med 1967; 276: 215-25.

2. Classification of hyperlipidaemias and hyperlipoproteinaemias. Bull World Health Organ 1970; 43: 891-915.

3. National Cholesterol Education Program (NCEP) Expert Panel on Detection, Evaluation, and Treatment of High Blood Cholesterol in Adults (Adult Treatment Panel III). Third Report of the National Cholesterol Education Program (NCEP) Expert Panel on Detection, Evaluation, and Treatment of High Blood Cholesterol in Adults (Adult Treatment Panel III) final report. Circulation 2002; 106: 3143-421.

4. Grundy SM, Stone NJ, Bailey AL, et al. 2018 AHA/ACC/ AACVPR/AAPA/ABC/ACPM/ADA/AGS/APhA/ASPC/NLA/ PCNA Guideline on the Management of Blood Cholesterol: Executive Summary: A Report of the American College of Cardiology/American Heart Association Task Force on Clinical Practice Guidelines [published online ahead of print November 8, 2018]. J Am Coll Cardiol November 2019; 73: 3168-209.

5. Catapano AL, Graham I, De Backer G, et al. 2016 ESC/ EAS guidelines for the management of dyslipidaemias. Eur Heart J 2016; 37: 2999-3058.

6. Banach $M$, Jankowski P, Jóźwiak J, et al. PoLA/CFPiP/PCS guidelines for the management of dyslipidaemias for family physicians 2016. Arch Med Sci 2017; 13: 1-45.

7. Phillips NR, Waters D, Havel RJ. Plasma lipoproteins and progression of coronary artery disease evaluated by angiography and clinical events. Circulation 1993; 88: 2762-70.

8. Nordestgaard BG, Tybjaerg-Hansen A. IDL, VLDL, chylomicrons and atherosclerosis. Eur J Epidemiol 1992; 8 Suppl 1: 92-8.
9. Miller M, Stone NJ, Ballantyne C, et al. Triglycerides and cardiovascular disease: a scientific statement from the American Heart Association. Circulation 2011; 123: 2292-333.

10. Gaudet D, Brisson D, Tremblay K, et al. Targeting APOC3 in the familial chylomicronemia syndrome. N Engl J Med 2014; 371: 2200-6.

11. Sampson UK, Fazio S, Linton MF. Residual cardiovascular risk despite optimal LDL cholesterol reduction with statins: the evidence, etiology, and therapeutic challenges. Curr Atheroscler Rep 2012; 14: 1-10.

12. Graham MJ, Lee RG, Brandt TA, et al. Cardiovascular and metabolic effects of ANGPTL3 antisense oligonucleotides. N Engl J Med 2017; 377: 222-32.

13. Farnier M. Future lipid-altering therapeutic options targeting residual cardiovascular risk. Curr Cardiol Rep 2016; 18: 65 .

14. Fredrickson DS. It's time to be practical. Circulation February 1975. http://www.ahajournals.org/doi/ abs/10.1161/01.cir.51.2.209. Accessed October 23, 2018.

15. Wood PD, Stern MP, Silvers A, Reaven GM, von der Groeben J. Prevalence of plasma lipoprotein abnormalities in a free-living population of the Central Valley, California. Circulation 1972; 45: 114-26.

16. Jones GJL, Hewitt D, Godin GJ, et al. Plasma lipoprotein levels and the prevalence of hyperlipoproteinemia in a Canadian working population. Can Med Assoc J 1980; 122: 37-46.

17. Gibson TC, Whorton EB. The prevalence of hyperlipidemia in a natural community. J Chronic Dis 1973; 26: 227-36.

18. Vergès B. Pathophysiology of diabetic dyslipidaemia: where are we? Diabetologia 2015; 58: 886-99.

19. Imga NN, Karci AC, Oztas D, Berker D, Guler S. Effects of vitamin D supplementation on insulin resistance and dyslipidemia in overweight and obese premenopausal women. Arch Med Sci 2019; 15: 598-606.

20. Stępień A, Stępień M, Wlazeł RN, Paradowski M, Banach $M$, Rysz J. Assessment of the relationship between lipid parameters and obesity indices in non-diabetic obese patients: a preliminary report. Med Sci Monit 2014; 20: 2683-8.

21. Sniderman A, Couture P, de Graaf J. Diagnosis and treatment of apolipoprotein B dyslipoproteinemias. Nat Rev Endocrinol 2010; 6: 335-46.

22. Holewijn S, Sniderman AD, den Heijer M, Swinkels DW, Stalenhoef AFH, de Graaf J. Application and validation of a diagnostic algorithm for the atherogenic apoB dyslipoproteinemias: ApoB dyslipoproteinemias in a Dutch population-based study. Eur J Clin Invest 2011; 41: 423-33.

23. Centers for Disease Control and Prevention. National Health and Nutrition Examination Survey: Sample Design, 2011-2014. https://wwwn.cdc.gov/nchs/data/series/sr02_162.pdf.

24. Martin SS, Blaha MJ, Toth PP, et al. Very large database of lipids: rationale and design. Clin Cardiol 2013; 36 641-8.

25. Centers for Disease Control and Prevention. National Health and Nutrition Examination Survey: Analytic Guidelines, 2011-2012. https://wwwn.cdc.gov/nchs/ data/nhanes/2011-2012/analytic_guidelines_11_12. pdf. Accessed October 24, 2018.

26. Martin SS, Blaha MJ, Elshazly MB, et al. Comparison of a novel method vs the friedewald equation for estimating low-density lipoprotein cholesterol levels from the standard lipid profile. JAMA 2013; 310: 2061-8. 
27. Centers for Disease Control and Prevention. Laboratory procedure manual. Apolipoprotein B. https://wwwn.cdc. gov/nchs/data/nhanes/2011-2012/labmethods/apob_g_met.pdf. Accessed October 24, 2018.

28. Centers for Disease Control and Prevention. National Health and Nutrition Examination Survey: LDL \& Triglycerides. https://wwwn.cdc.gov/Nchs/Nhanes/20112012/TRIGLY_G.htm. Accessed October 24, 2018.

29. Schaefer JR. Unraveling hyperlipidemia type III (dysbetalipoproteinemia), slowly. Eur J Hum Genet 2009; 17: 541-2.

30. Hopkins PN, Brinton EA, Nanjee MN. Hyperlipoproteinemia type 3: the forgotten phenotype. Curr Atheroscler Rep 2014; 16: 440.

31. de Ferranti SD, Rodday AM, Mendelson MM, Wong JB, Leslie LK, Sheldrick RC. Prevalence of familial hypercholesterolemia in the 1999 to 2012 United States National Health and Nutrition Examination Surveys (NHANES). Circulation 2016; 133: 1067-72.

32. Khera AV, Won HH, Peloso GM, et al. Diagnostic yield and clinical utility of sequencing familial hypercholesterolemia genes in patients with severe hypercholesterolemia. J Am Coll Cardiol 2016; 67: 2578-89.

33. Fredrickson DS, Lees RS. A system for phenotyping hyperlipoproteinemia. Circulation 1965; 31: 321-7.

34. Grundy SM, Mok HY, Zech L, Steinberg D, Berman M. Transport of very low density lipoprotein triglycerides in varying degrees of obesity and hypertriglyceridemia. J Clin Invest 1979; 63: 1274-83.

35. Menke A, Casagrande S, Geiss L, Cowie CC. Prevalence of and trends in diabetes among adults in the United States, 1988-2012. JAMA 2015; 314: 1021-9.

36. Albuquerque EMV, de Faria EC, Oliveira HCF, Magro DO, Castilho LN. High frequency of Fredrickson's phenotypes IV and IIb in Brazilians infected by human immunodeficiency virus. BMC Infect Dis 2005; 5: 47.

37. Adedinsewo D, Taka N, Agasthi P, Sachdeva R, Rust G, Onwuanyi A. Prevalence and factors associated with statin use among a nationally representative sample of us adults: National Health and Nutrition Examination Survey, 2011-2012. Clin Cardiol 2016; 39: 491-6.

38. Sacks FM, Alaupovic P, Moye LA, et al. VLDL, apolipoproteins $\mathrm{B}, \mathrm{CIII}$, and $\mathrm{E}$, and risk of recurrent coronary events in the Cholesterol and Recurrent Events (CARE) trial. Circulation 2000; 102: 1886-92.

39. Hodis HN. Triglyceride-rich lipoprotein remnant particles and risk of atherosclerosis. Circulation 1999; 99 : 2852-4.

40. Toth PP, Thanassoulis G, Williams K, Furberg CD, Sniderman A. The risk-benefit paradigm vs the causal exposure paradigm: LDL as a primary cause of vascular disease. J Clin Lipidol 2014; 8: 594-605.

41. Civeira F, Pocoví M, Cenarro A, et al. Apo E variants in patients with type III hyperlipoproteinemia. Atherosclerosis 1996; 127: 273-82.

42. Baggio G, Manzato E, Gabelli C, et al. Apolipoprotein C-II deficiency syndrome. Clinical features, lipoprotein characterization, lipase activity, and correction of hypertriglyceridemia after apolipoprotein C-II administration in two affected patients. J Clin Invest 1986; 77: 520-7.

43. Gaudet D, Alexander VJ, Baker BF, et al. Antisense inhibition of apolipoprotein C-III in patients with hypertriglyceridemia. N Engl J Med 2015; 373: 438-47. 